\title{
Effect of CB-154 Therapy on the Glucose Tolerance, IRI and GH Response to Glucose Administration in Acromegaly
}

\author{
Reiko DEMURA, Hajime YAMAGUCHI, Hiroshi DEMURA \\ and Kazuo SHIZUME \\ Department of Internal Medicine, Tokyo Women's Medical \\ College, Shinjuku-ku, Tokyo 162, Japan
}

\begin{abstract}
Synopsis
Effects of CB-154 therapy on the glucose tolerance, IRI and GH responses to the oral glucose administration in 5 acromegalic patients with impaired glucose tolerance were examined. Glucose tolerance improved in all patients with a reduction in $\mathrm{GH}$ levels. Insulin secretion in response to the oral glucose administration was exaggerated in 3 patients, while it was suppressed in 2 patients who had severely impaired glucose tolerance. After receiving CB-154 therapy, hyperresponsiveness of insulin observed in the 3 patients and a suppressed response in one patient were corrected to normal, while a low response in another stayed unchanged. This observation suggest that variable abnormalities in insulin secretion induced by the excess of GH improve with a decrease in plasma GH levels. However, the abnormal GH secretion in response to hyperglycemia persisted still after administration of CB-154 in spite of decreased basal plasma GH levels.
\end{abstract}

An improvement in glucose tolerance or in the clinical condition of diabetic patients due to acromegaly during CB-154 therapy has been noted by many investigators such as Thorner et al. (1975), Sachdev et al. (1975), Cassar et al. (1976), Benker et al. (1976), Belforte et al. (1977) and Wass et al. (1977). Though it has been confirmed that the levels of blood glucose and plasma growth hormone $(\mathrm{GH})$ were reduced in most of the acromegalic patients after administration of CB-154, the data concerning immunoreactive insulin (IRI) and GH in response to glucose load during CB-154 therapy have been scarce and inconsistent. We have examined the effect of CB-154 therapy on blood glucose, IRI and GH responses to the oral glucose load, and found that the glucose tolerance as well as insulin secre-

Received November 4, 1977. tion, which was variable before the therapy, improves with a decrease in basal plasma $\mathrm{GH}$ levels. However, abnormal GH response to glucose load persisted still after the CB154 therapy in spite of decreased plasma basal GH levels.

\section{Materials and Methods}

Five patients with acromegaly who had been treated with CB-154 for at least 4 weeks were subjected to the studies (Table 1). Glucose tolerance

Table 1. Patients subjected to the study.

\begin{tabular}{ccccc}
\hline case & age & sex & $\begin{array}{c}\text { CB-154 } \\
\text { dose }\end{array}$ & $\begin{array}{c}\text { duration } \\
\text { (months) }\end{array}$ \\
\hline 1 & 56 & $\mathrm{~m}$ & 2.5 & 7 \\
2 & 53 & $\mathrm{~m}$ & 5.0 & 12 \\
3 & 61 & $\mathrm{f}$ & 40 & 2 \\
4 & 26 & $\mathrm{~m}$ & 20 & 3 \\
5 & 41 & $\mathrm{~m}$ & 10 & 2 \\
\hline
\end{tabular}


test (GTT) was performed before and 4 weeks after CB-154 therapy by the oral administration of $100 \mathrm{~g}$ of glucose in the morning after their overnight fast. Blood glucose, plasma IRI and GH were measured in the same sample at 30-min intervals for $180 \mathrm{~min}$. Blood glucose was measured by glucose oxidation method using the autoanalyzer, and plasma IRI and GH were measured by the RIA kits marketed by Dainabot in Japan.

Normal values for fasting blood glucose were $83 \pm$ $12 \mathrm{mg} / \mathrm{d} l$. Glucose tolerance was defined as impaired when blood glucose at 60 and $120 \mathrm{~min}$ were more than 140 and $110 \mathrm{mg} / \mathrm{d} l$, respectively, according to the diagnostic criteria of the Japan Diabetic Society. The basal serum IRI and maximal IRI response to oral administration of $100 \mathrm{~g}$ glucose in normal subjects under 50 years of age were $10.5 \pm 1.4$ and $86.4 \pm 7.7$ $\mu \mathrm{U} / \mathrm{ml}$, respectively, while those in normal subjects older than 50 years of age were $7.8 \pm 1.0$ and $73.5 \pm$ $10.1 \mu \mathrm{U} / \mathrm{ml}$, respectively in our laboratory (Hagura, 1969).

\section{Results}

Glucose tolerance before and after the CB-154 is shown in the top column of Fig. 1. Glucose tolerance in all of the patients were abnormal before the therapy. One patient (case 2) has had overt clinical diabetes and has been treated with insulin. After the CB-154 therapy, a decrease in fasting blood glucose was observed and glucose tolerance improved in various degrees in all of the patients. As shown in Fig. 2, the insulin requirement could be reduced in a large extent and urinary glucose disappeared after administration of CB154 in case 2.
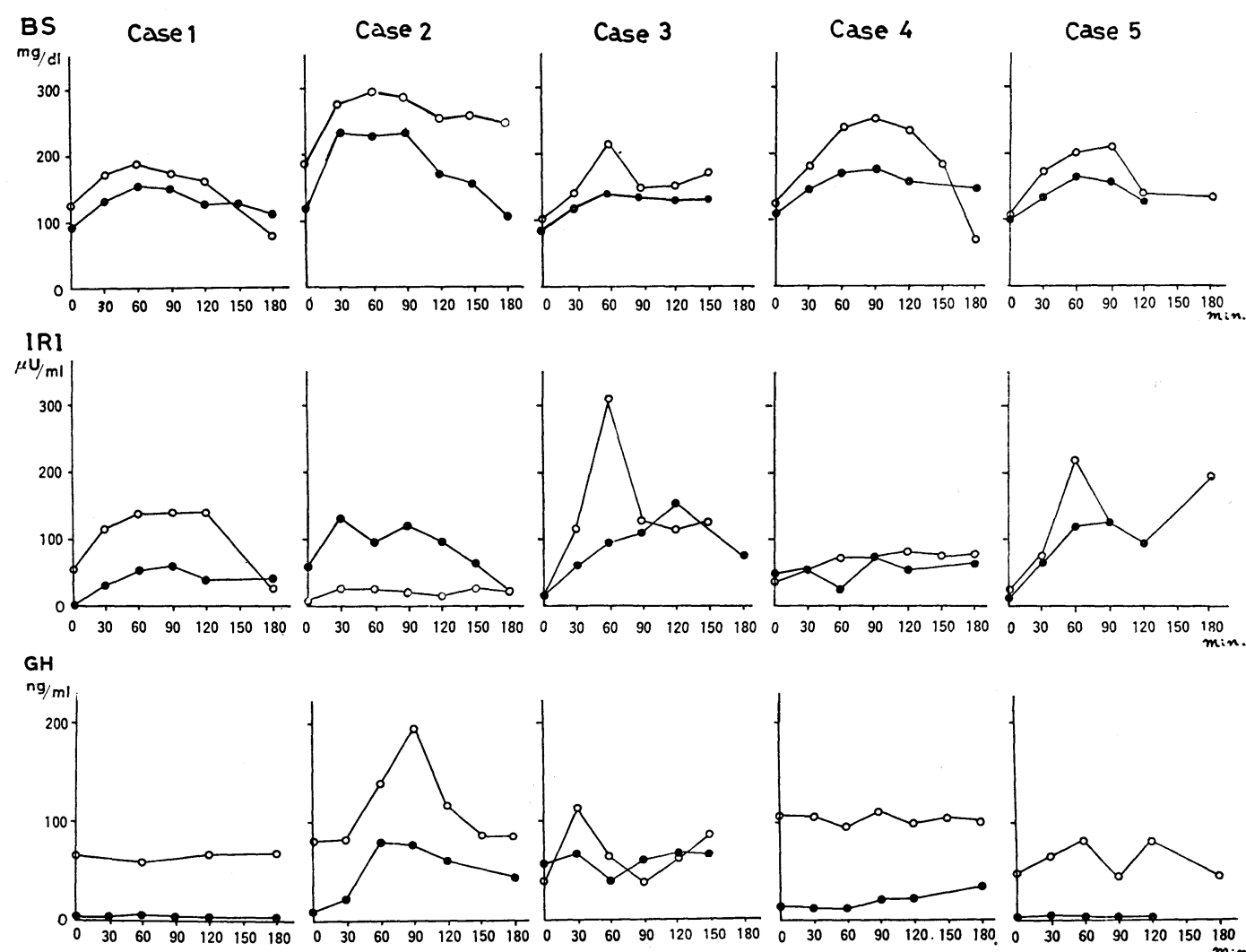

Fig. 1. Responses of blood glucose (top), plasma IRI (middle) and GH (bottom) to glucose administration before and after the CB-154 therapy. O-O indicates before the therapy and 
Plasma IRI responses to glucose load are shown in the middle column of Fig. 1. Plasma IRI showed hyperresponsiveness to GTT in 3 among the 5 patients (case 1, 3 and 5) with a slightly elevated basal level in one of them (case 1). On the contrary, the response of plasma IRI was suppressed in the remaining 2 patients (case 2 and 4 ) with especially poor glucose tolerance. The basal IRI level was low in one of them (case 2), while it was normal in the other (case 4). After receiving CB-154 therapy, the IRI responses in the former 3 patients were reduced in some extent with a decrease in the basal IRI level in case 1, though the responses still remained exaggerated in case 3 and 5 . On the contrary, the IRI response became potentiated with an elevation of the basal level of IRI after the therapy in case 2, who showed a suppressed response before the therapy. The IRI response in the remaining patient (case 4) with an initial low response stayed essentially the same.

Plasma GH responses to oral administration of glucose before and after the CB154 are shown in the bottom column of Fig. 1. Plasma GH levels were elevated in all of the patients and no decline in response to hyperglycemia was noted. The paradoxical rise by more than $100 \%$ above the basal level was noted in 2 patients. Abnormal patterns of $\mathrm{GH}$ response remained essentially the same in all of the patients, though a remarkable reduction in the basal GH level was observed after adminstration of CB-154 except for one (case 3) in whom a diminished increment in a paradoxical rise was noted.

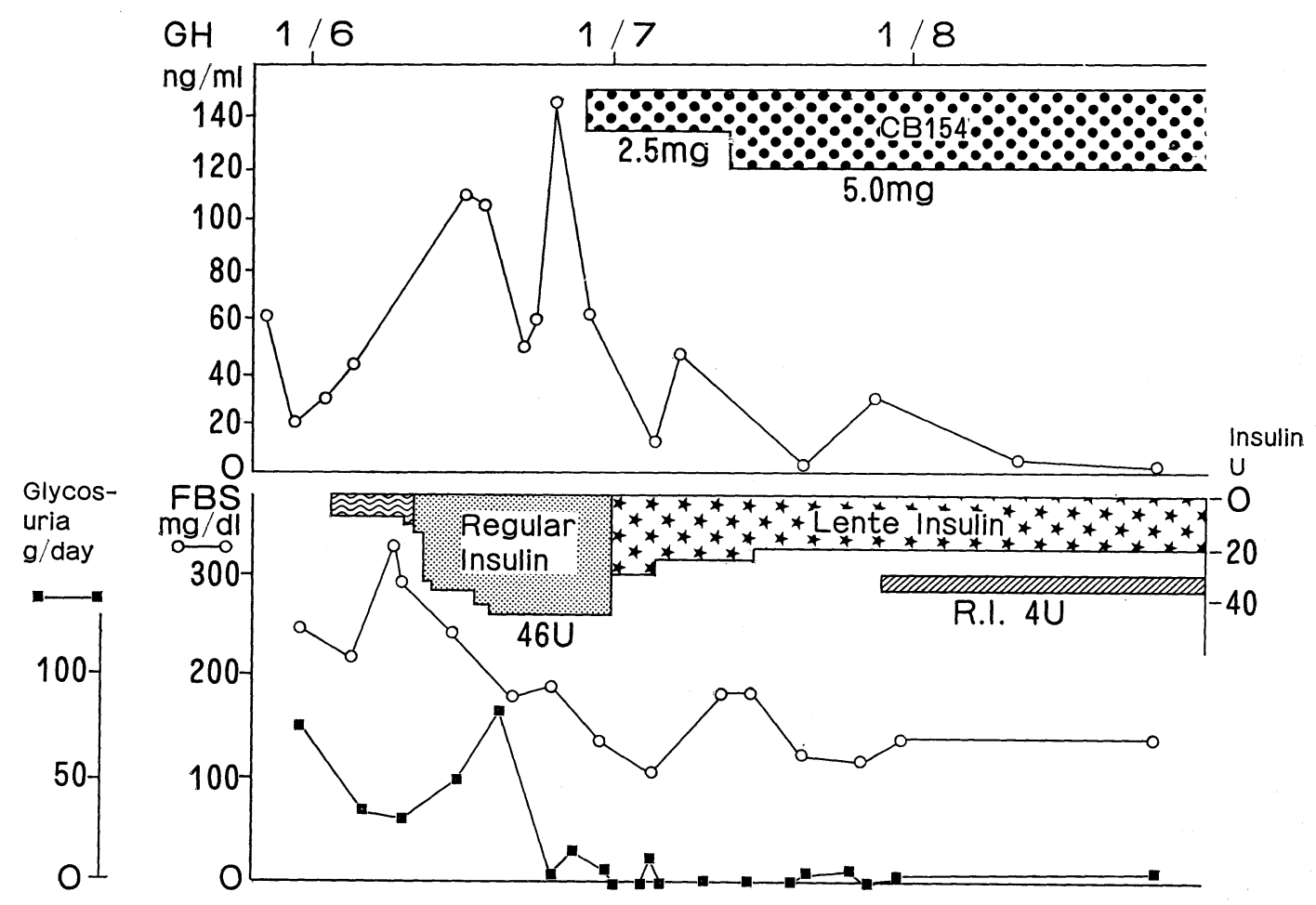

Fig. 2. Clinical course of diabetes mellitus in case 2 before and after the CB-154 therapy. 


\section{Discussion}

The present studies demonstrated that glucose tolerance improved in various degrees shortly after the CB-154 therapy in acromegalic patients with a reduction in the basal GH levels.

An impaired carbohydrate metabolism has been known to be one of the major metabolic abnormalities associated with acromegaly. The excess of $\mathrm{GH}$ has been proposed as a cause for impaired carbohydrate metabolism by its antagonizing action to insulin at the level of glucose uptake and utilization as shown by Riddick et al. (1962). However, recent studies by Cerasi et al. (1964), Beck et al. (1965), and Okuyama et al. (1977) demonstrated that an impaired carbohydrate metabolism associated with acromegaly was multifactorial, mainly based on the observation that insulin secretion in response to the glucose load in acromegalic patients were variable. Insulin secretion in some acromegalic patients with overt diabetes mellitus was reported to be diminished, but it was rather exaggerated in most of the acromegalic patients with various degrees of impaired glucose tolerance. As suggested by Beck et al. (1965), an increased insulin response may be in part a result of hyperglycemia induced by the excess of $\mathrm{GH}$, but it may also reflect an increased insulin-secreting capacity of the pancreas. However, the insulin-secreting capacity may decrease with a progress of diabetes mellitus through the impaired islet cell function.

Variability in the insulin response to oral glucose load observed before the CB154 therapy in the present studies could be explained in this connection. Patients with a mild hyperglycemia exhibited hyperresponsiveness of IRI, while those with a marked hyperglycemia or overt diabetes mellitus showed a poor response of insulin to oral glucose load. Correction of insulin secretion in 4 out of 5 patients towards normal after the CB-154 therapy suggested that the abnormalities in the insulin secretion observed before the therapy were caused by the excess of $\mathrm{GH}$ and readily reversible when plasma GH levels were reduced. It could not deny the presence of primary diabetes mellitus in a patient (case 4) who had a poor insulin response throughout the studies.

There have been a few reports concerning the insulin response after the CB-154 therapy for acromegalic patients. Thorner et al. (1975) reported no change, while Sachdev et al. (1975) and Belforte et al. (1977) reported a suppression in the insulin secretion after the administration of CB-154. These inconsistencies may be partly due to the variability in insulin secretion in acromegalic patients before the therapy.

In addition, the present studies demonstrated that the abnormal GH response to oral glucose load persisted still after the CB154 therapy. This was in accord with the previous results by Thorner et al. (1975), Cassar et al. (1976), Benker et al. (1976) and Wass et al. (1977), in which the basic patterns of $\mathrm{GH}$ response to the oral glucose load were reported to be unchanged, though the paradoxical rise was abolished in some of the patients. This suggested that the pathognomonic abnormalities in the GH secretion in acromegalic patients remained unchanged even with a marked reduction in the basal GH levels.

In conclusion, CB-154 is an effective GH-lowering agent, which accompanied with an improvement in the glucose tolerance and insulin secretion to oral glucose load in acromegalic patients. 


\section{References}

Beck, P., D. S. Schalch, M. L. Parker, D. M. Kipnis and W. H. Daughaday (1965). J. Lab. Clin. Med., $66,366$.

Belforte, L., F. Camanni, P. G. Chiodini, A. Liuzzi, F. Massara, G. M. Molinatti, E. E. Müller and F. Silvestrini (1977). Acta Endocrinol. (Kbh.) 85, 235.

Benker, G., W. Zäh, K. Hackenberg, B. Hamburger, H. Günnewig and D. Reinwein (1976). Horm. Metab. Res. 8, 291.

Cassar, J., K. Mashiter, G. Sullivan and G. F. Joplin (1976). Acta Endocrinol. (Kbh.) 82, 39.

Cerasi, E. and R. Luft (1964). Lancet 2, 709.
Okuyama, M. and K. Yasuda (1977). J. Jap. Soc. Intern. Med. 66, 22. (In Japanese)

Hagura, R. (1969). J. Tokyo Women's Med. Coll. 39, 418.

Riddick, F. A., D. M. Reisler and D. M. Kipnis (1962). Diabetes 11, 171.

Sachdev, Y., A. Gomez-Pan, W. M. G. Tunbridge, A. Duns, D. R. Weightman, R. Hall and S. K. Goolamali (1975). Lancet 13, 1164.

Thorner, M. O., A. Chait, M. Aitken, G. Benker, S. R. Bloom, C. H. Mortimer, P. Sanders, A. S. Mason and G. M. Besser (1975). Br. Med. J. 1, 299.

Wass, J. A. H., M. O. Thorner, D. V. Morris, L. H. Rees, A. S. Mason, A. E. Jones and G. M. Besser (1977). Br. Med. J. 1, 875. 